Int. J. Dev. Biol. 48: 343-347 (2004)

Short Communication

\title{
Dynamics of decapentaplegic expression during regeneration of the Drosophila melanogaster wing imaginal disc
}

\author{
JAAKKO MATTILA*,1, LEONID OMELYANCHUK ${ }^{2}$ and SEPPO NOKKALA ${ }^{1}$ \\ ${ }^{1}$ Laboratory of Genetics, Department of Biology, University of Turku, Finland and ${ }^{2}$ Laboratory of Cell Cycle Genetics, Institute of Cytology \\ and Genetics, Lavrent'eva 10, Novosibirsk, Russia
}

\begin{abstract}
Regeneration of an imaginal disc involves highly ordered proliferation and pattern regulation of the newly formed tissue. Although the general principles of imaginal disc regeneration have been extensively studied, knowledge of the underlying molecular mechanisms is far from complete. Results from other model organisms suggest that regeneration is the result of local recapitulation of the normal patterning genes. To analyze the dynamics of one major Drosophila patterning gene, decapentaplegic (dpp), in wing imaginal disc regeneration, a vital GFP reporter together with iontophoretic cell labeling were used. Our observations reveal that the restoration of compartment-border-specific dpp expression is a common event in imaginal disc regeneration. However, we did not find evidence of an upregulation of dpp expression during the regeneration process.
\end{abstract}

KEY WORDS: Drosophila melanogaster, wing imaginal disc, regeneration, decapentaplegic

The development of Drosophila imaginal discs begins from a small group of similar cells at the blastoderm stage and results in the formation of adult appendages consisting of diverse cell types. This transformation is known to involve highly ordered cell proliferation and differentiation, and is shown to be under the control of diffusible patterning molecules, morphogens. One such protein, Decapentaplegic (Dpp), acts as a long-range morphogen, emanating from the vicinity of anterior-posterior $(A / P)$-compartment border, patterning the developing wing field (reviewed by Podos and Ferguson, 1999). The fundamental role of Dpp in controlling proliferation and pattern formation of the wing disc has been shown in various experiments (e.g. Chen and Struhl, 1996, Lecuit and Cohen, 1998).

The Drosophila imaginal discs have been well-studied models of epimorphic regeneration. Although the process has been extensively studied in tissue and cell level, little is known about the underlying molecular interactions controlling pattern regulation in imaginal discs. However, recent molecular data on regeneration in other model organisms has revealed many interesting properties of regeneration in general. For example, in urodele (Tanaka, 2003) and frog tadpole (Beck et al., 2003) tail regeneration, the process can be understood by a local recapitulation of the normal developmental events. This means that major patterning genes are reemployed in the blastema region where extensive cell proliferation and pattern regulation occurs.

Although expressions of early patterning genes in the blastema of Drosophila imaginal discs have not been demonstrated to date, some evidence exists that regeneration alters the $d p p$ gene expression. Milétich and Limbourg-Bouchon (2000) have observed that imaginal discs bearing $s / m b$ clones induce widening of the $A$ / $\mathrm{P}$ compartment-border-specific dpp expression. The authors proposed that the widening could be a reaction to the regeneration process produced by the s/mb-induced cell death. Similar widening of the dppexpression has also been seen when cell death has been induced by X-irradiation (Milétich and Limbourg-Bouchon, 2000), or by the use of a temperature-sensitive cell-lethal allele of suppressor of forked (Brook et al., 1993). These results suggest that $d p p$ is one of the genes involved in the regulation of imaginal disc regeneration and that the control of pattern regulation does not solely depend on the local interaction of cells apposed by wound healing as suggested by the polar co-ordinate model (French et al., 1976).

In addition to the deviations observed in the compartmentborder-specific dpp expression, Brook et al. (1993) found that enhancer trap line E32, which is thought to reflect the expression of the dppgene, is expressed in the blastema of a fragmented wing imaginal disc. This was inferred as a requirement of the dppgene in the control of the regeneration process. However, the occurrence and specific dynamics, as well as the function of the blastema specific dpp expression were left unresolved.

Abbreviations used in this paper: dpp, decapentaplegic; GFP, green fluorescent protein.

\footnotetext{
*Address correspondence to: Jaakko Mattila. Laboratory of Genetics, Department of Biology, University of Turku, FIN-20014 Turku, Finland. Fax: +358-2-333-6680. e-mail:jailma@utu.fi
} 
In this study we examined the dynamics and occurrence of ectopic $d p p$ expression in fragmented and in vivo cultured wing imaginal discs. We present evidence that the restoration of the compartment-border-specific dpp expression is a common event in Drosophila wing disc regeneration. It is shown that dppexpression is restricted only to blastemas which originate from the interaction of two different compartments, namely anterior and posterior. Further, our results suggest that the widening of the compartment-border-specific dppexpression is not induced by the regeneration process, at least to the extent detectable by our method.

\section{Results and Discussion}

The in vivoculture of Drosophilaimaginal discs has proven to be a powerful method in the field of regeneration (O'Brochta \& Bryant 1987, Bryant \& Fraser 1988, Gibson \& Schubiger 1999). Many properties of pattern regulation, now widely accepted, have been revealed by the culturing of imaginal disc fragments in adult and larva abdomens. In this study, we have employed in vivoculturing of disc fragments in adult hosts, together with iontophoretic cell labeling (Fig. 1). To test our culture conditions, we localized the area of regenerative proliferation with the $\mathrm{H} 3$ mitosis marker and found that it coincided perfectly with the site of removed tissue (Fig. 2). The uncut control discs cultured under the same conditions showed a significantly reduced mitosis number without any preferable concentrations of mitotic cells. It is therefore reasonable to believe that the culture method utilized is able to uncover the true genetic interactions involved in regeneration.

The iontophoretic cell labeling was tested for stability of the labeled spots during the in vivoculture (Fig. 3). The labeling pattern was clearly visible after two days of culture, although some transformations in the shape and label intensity could be seen. We concluded that these alterations were due to the proliferation of the labeled cells or those nearby. However, this control showed that the position of the labeled spots was constant and, therefore, the control spots can be used as reference points for the compartmentborder-specific dpp expression.

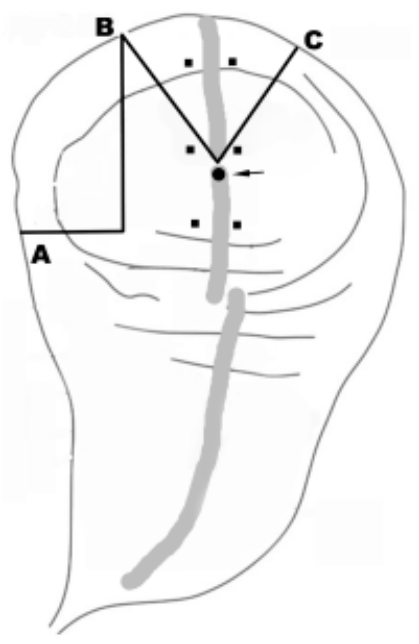

Fig. 1. The incisions of the imaginal disc. The control cut $A B$ was used together with rhodamine cell labeling (black squares) on either anterior or posterior, or both sides of dpp expression. The number of the marked cells was restricted by time; the discs could be kept in inappropriate culture condition (i.e. Hank's solution) for only a short period of time (in our experiment for approximately 20 min). The BC cut was used together with rhodamine cell labeling (black circle denoted by the arrow) in the location of disturbed

$\mathrm{dpp}$ expression. The gray stripe running through the length of the disc denotes compartment-border-specific dpp expression. The disc is orientated anterior side to the left and ventral up as in all the illustrated discs.

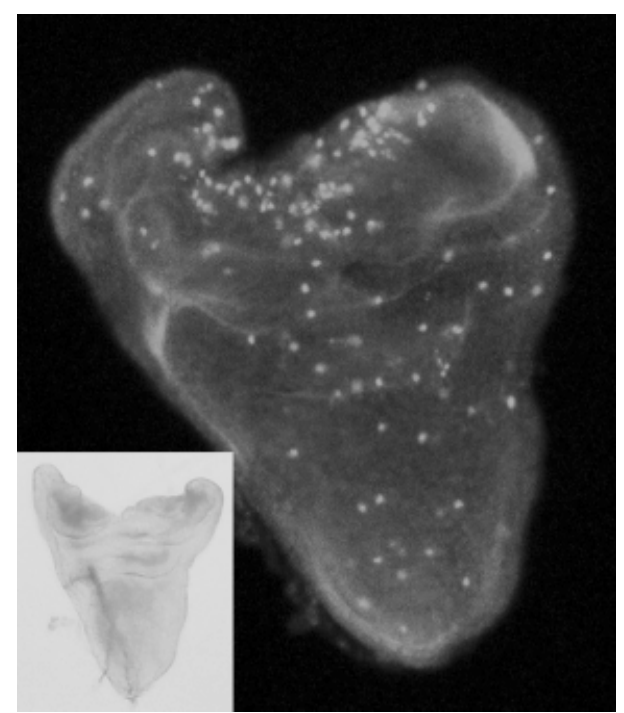

Fig. 2. Control of culture conditions. The inserted picture shows a wing imaginal disc after four days of in vivo culturing. After this culture period, developmental proliferation has ceased. The disc was cut (incision BC in Fig. 1) in order to stimulate regenerative proliferation. In the large picture, the same disc can be seen labeled with the H3 mitosis marker after an additional 48 hours of culturing. The area of the healing wound shows extensive labeling compared to the rest of the disc.

The results of Milétich and Limbourg-Bouchon (2000) showed that imaginal discs bearing $s / m b$ clones broaden the A/P -compartment-border-specific $d p p$ expression. Such a widening was inferred to result from the regeneration process since a similar effect was also seen when cell death was induced using $X$ irradiation (Milétich and Limbourg-Bouchon, 2000) or by the use of a temperature-sensitive cell-lethal allele of suppressor of forked (Brook et al., 1993). We were interested to see whether the widening could also be induced by incision of the disc, thus providing evidence for $d p p^{\prime}$ 's role in the control of the local regeneration process. In order to do so, we have utilized the excision $A^{\prime} B$, where only tissue of the $A$ compartment was extirpated and the cells expressing the $d p p$ gene were left intact.

To obtain firm evidence for the widening of $d p p$ expression, i.e. recruitment of new cells to $d p p$-expressing state resulting from the disc fragmentation, we expected the GFP domain to move closer or to even overlap the rhodamine-labeled cells. From all the $d p p$ patterns resulting from the A'B cut we found that $81 \%(35 / 43)$ of discs had an expression pattern indistinguishable from the one before culturing (Fig. 4 A,B). The remaining 19\% showed deviations of the GFP domain exclusively in the wing pouch (an example is given in Fig. 4D). However, none of these discs were observed to possess the overlap of the GFP expression and rhodamine labeled spots. The observed "widening" deviations in the GFP pattern (as in Fig. 4D) could be explained by the distorted disc morphology caused by wound healing stretching the wing pouch tissue. In our experiment wound healing was sometimes seen to cause transient aberrant tissue folding in the wing pouch, predominantly in the vicinity of the wound, resulting in reorganization of the disc epithelia. In such cases the localization of the GFP expression is no longer reliable. As a result we conclude that although we were unable to prove widening of the $d p p$ expression in regard to the rhodamine-labeled cells (Fig. 4) we could not exclude the possibil- 


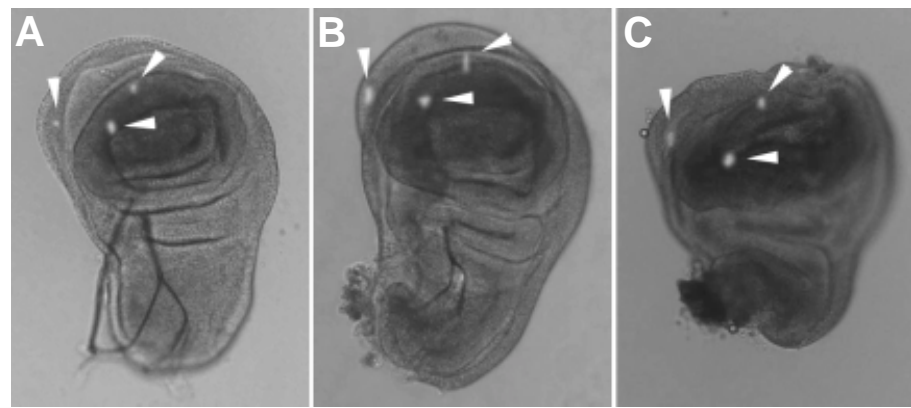

Fig. 3 (Left). Control iontophoresis experiment showing the constant pattern of cell labeling. (A) An intact wild type wing imaginal disc labeled by iontophoresis. The same disc after (B) 24 and (C) 48 hours of in vivo culture. Arrowheads mark the labeled cells in (A), (B) and (C).
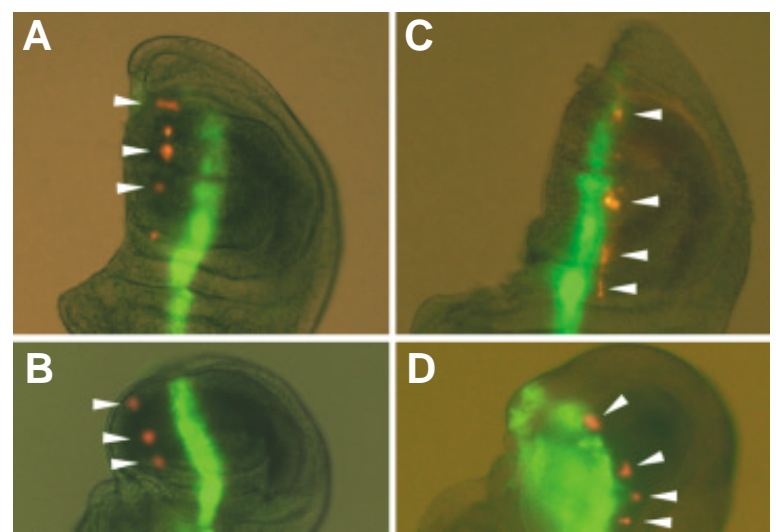

Fig. 4 (Right). Expression of the dpp gene resulting from the AB cut (see Fig. 1). (A) The incised wing imaginal disc was rhodamine-labeled anteriorly to the dpp expression zone (arrowheads). (B) After 48 hours of in vivo culturing, the expression zone was not altered with regard to the rhodamine spots. Note the two missing rhodamine spots in (B). These spots represent either incorrect needle positioning causing the dye to be localized in the extracellular matrix or insufficient volume of the dye. (C) Another disc labeled posteriorly to the dpp expression zone. (D) After 24 hours of in vivo culturing, the GFP domain extends in the direction of the wound (see text for interpretation of such an event). There is no indication of the region of expression broadening towards the rhodamine labeled cells.

ity that the widening of the dppexpression is rarely induced by the fragmentation and therefore left undiscovered in our experiment. Interestingly, the widening of the compartment-border-specific $d p p$ expression has also been reported with fat as well as fat $^{18}$ overgrowth mutant alleles (Garoia etal., 2000). The latter suggests that extensive dppexpression is related to proliferation rather than regeneration per se. It is also worth noting that the cell deaths induced during the course of the normal growth period, as done in Milétich and Limbourg-Bouchon (2000) and Brook etal. (1993), are likely to delay the disc development. Such a developmental retardation could result in prolonged existence of the wide dppdomain characteristic of the early third instar wing disc (Burke and Basler 1996). As we failed to establish the upregulation of dppexpression in our experiment, we hypothesize that regenerative proliferation in the blastema is controlled by other mechanisms, independent of the diffusing DPP morphogen from the A/P compartment border.

Incision A'B showed no detectable dppexpression in the wound edges or the blastema during the observed culture period. This is in agreement with the known imaginal disc development; $d p p$ expression is induced by the Hedgehog $(\mathrm{Hh})$ morphogen diffusing from the posterior compartment. The lack of $d p p$ expression can also be interpreted in regard to the signaling pathways controlling the wound healing process. The involvement of the JNK signaling pathway orchestrating the actin dynamics necessary for cell sheet movement is well characterized in dorsal closure (Noselli, 1996) and adult cuticle wound healing (Rämet et al., 2002). We have observed similar activation of JNK signaling also in the initial stages of wing imaginal disc wound healing (unpublished results). Glise and Noselli (1997) showed that JNK signaling in the leading edge cells of dorsal closure induces the Dpp/TGF signaling pathway. Although the specific function of this pathway in dorsal closure is unknown, it has been proposed that Dpp controls the motility and cell shape changes of the lateral epithelia (Riesgo-Escovar and Hafen, 1997). In adult cuticle wound healing such Dpp signaling has not been found (Rämet et al., 2002). The absence of dpp expression in the wound edges produced by the incision $A^{\prime} B$ suggests that the imaginal disc healing process shows closer genetic similarity to adult cuticle wound healing than dorsal closure.

To investigate the possible dppexpression in blastema originating from different compartments the incision B' $\mathrm{C}$ was used. The cut removed the ventral portion of the $\mathrm{A} / \mathrm{P}$-compartment border and $\mathrm{a}$ rhodamine-labeled cell was introduced to mark the distal end of the remaining $d p p$ expression (Fig. 1). The labeled cell served as a reference point, whereby it was possible to distinguish between the dppexpression before and after culturing. The observed temporal (Fig. 5) and spatial (Fig. 6) dynamics of dppexpression during the regeneration process indicated that $d p p$ induction in the $\mathrm{B}^{\prime} \mathrm{C}$
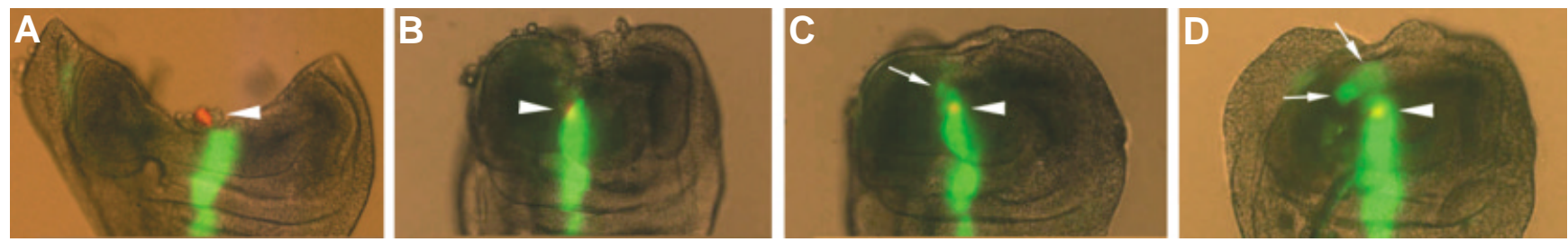

Fig. 5. A/P compartment border restoration during regeneration. (A) A wing imaginal disc before in vivo culturing showing the rhodamine-labeled cell marking the rest of the dpp expressing tissue (arrowhead denoting the labeled cell in (A), (B), (C) and (D)). (B) The same disc after 48 hours of in vivo culturing. Wound healing has brought the wound edges together but the labeled cell shows that dpp expression has not yet been restored. (C) The same disc after 72 hours of culturing. Some new dpp expression can be seen in the area of the regenerating blastema (arrow). (D) The same disc after 96 hours of culturing. The dpp expressing zone has extended toward the disc edge (arrows). 

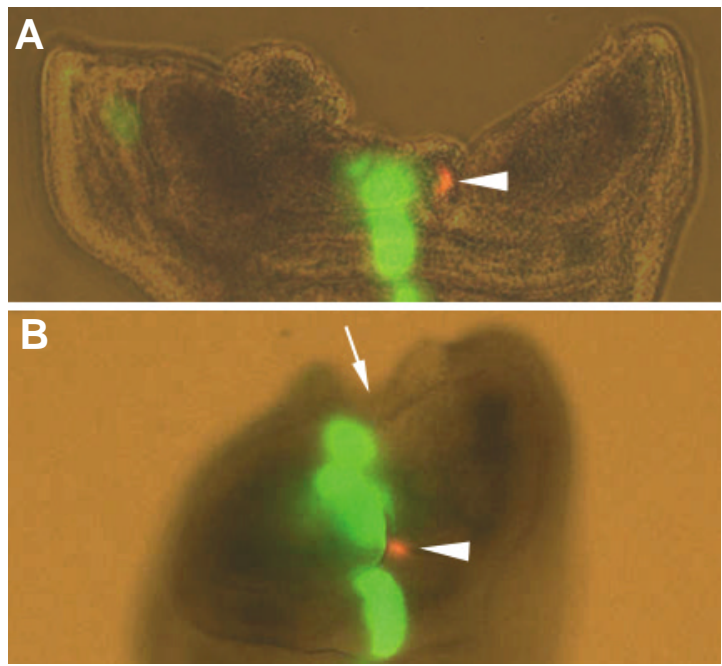

Fig. 6. Compartment-border-specific dpp expression is restored to the cells of the anterior compartment. (A) A wing imaginal disc before culturing. (B) The same disc after 96 hours of in vivo culturing. The arrow marks the site of wound healing which can be deduced from the "notch" in the wing margin. Novel dpp expression can be distinguished to be localized anteriorly from the site of the "notch". Arrowheads denote rhodamine-labeled cells in (A) and (B).

blastema was a common event. Our results showed that new $d p p$ expression emerges always $(\mathrm{N}=26)$ in the anterior side of the healed wound (Fig. 6). This is in agreement with the normal disc development since only the anterior cells are responsive to the $\mathrm{Hh}$ signal diffusing from the posterior side (Strigini and Cohen, 1997). If the appearance of novel dppexpression were indeed to follow the course of normal development, being dependent on the signal from the posterior compartment, one should not detect any expression before the ability of the wound edges to exchange information has been restored. Bryant and Fraser (1988) have studied the movement of intracellular dye molecules between imaginal disc wound edges and showed that only after three days the gap-junctional communication is fully re-established. The temporal dynamics of dpp expression resulting from the $\mathrm{B}^{\prime} \mathrm{C}$ cut demonstrate that new dppexpression becomes visible after three days of culturing in the apex of the healed wound and gradually grows distally during the next 24 hours (Fig. 5) eventually covering the whole length of the wound (Fig. 6). This kind of dynamic supports the view of $d p p$ expression being a consequence of the exchange of information between the cells of two different compartments.

Despite the importance of the compartment-border-specific gene expression during normal development, a complete imaginal disc can be restored from a tissue of only one compartment by the regeneration process (Bryant, 1975). This observation implies that compartment borders can reappear during regeneration. More straightforward evidence for the process was presented by Kunieda et al. (1997). The authors demonstrated that the activation of the compartment-border-specific genes can take place during the regeneration of a Sarcophaga peregrine leg disc. In addition, a recent study by Gibson and Schubiger (1999) showed that a new compartment border is activated in a duplicating Drosophila leg disc. Our results support the inference made by Kunieda et al. (1997) that the compartment borders are restored during the regeneration process. However, the temporal progression of the border restoration differs between the studies; in Kunieda et al. (1997) wingless-expression was detected only after 2 days of culture. The most probable explanation is that this is due to the differences in the experimental approaches rather than in the fundamental cellular processes; in our study, the cultivation of the incised discs was carried out in adult female abdomens instead of using artificial in vitro cultivation. As discussed in Kunieda et al. (1997), the concentration of ecdysone, among other additives, is probably a crucial determinant of wound healing in vitro, and consequently the border restoration progression.

Ectopic expression in the blastema of the fragmented wing imaginal disc was also observed in the E32 enhancer trap insertion near the $d p p$ gene and was interpreted as reflecting an additional requirement for $d p p$ in the regeneration process (Brook et al., 1993). The vital GFP reporter of dppexpression used in our study revealed expression only when the wound edges originated from different compartments, namely anterior and posterior, and as also noted by Brook et al. (1993), is only induced on the anterior side of the healing wound. Our finding suggests that $d p p$ is not a regeneration gene per se, but the expression is a secondary effect from the apposition of cells with different selector gene identity.

The present study demonstrates that the blastema specific $d p p$ expression is dependent on the compartmental identity of the wound edge cells. The expression emerges only from the apposition of anterior and posterior cells. This can be interpreted as a restoration of the wing disc A/P compartment-border signaling which most likely recapitulates the interactions governing the normal disc development. However, our results do not support the view that compartment-border-specific $d p p$ expression is upregulated in regenerating Drosophilawing disc.

\section{Experimental Procedures}

\section{Visualization of dpp expression and iontophoresis}

The visualization of the $d p p$ expression in wing imaginal discs was conducted using a vital GFP reporter. A cross of $\mathrm{P}\{$ Gal4-dpp.blk1\}/TM6B, $T b$ (obtained from $S$. Cohen) individuals with individuals bearing $y w$, $\mathrm{P}\left\{w^{+} C=\right.$ UAS-GFP.S65T $\}$ (insertion in II chromosome, obtained from $\mathrm{C}$. Lehner) was performed and imaginal discs were dissected from non- Tb wandering-stage larvae. Although lacking the ability to reliably score the deviations in gene expression intensity, the reporter allowed study of the dynamics of expression in the same imaginal disc. Also, the use of iontophoresis restricted us to using visualization methods where tissue was left unfixed.

To prevent false interpretation of gene expression caused by tissue reorganization due to the wound healing process we introduced "reference" cells near the $d p p$ expression by labeling them with dextran-conjugated rhodamine (Molecular Probes) (Fig. 1). The labeling was performed by iontophoresis modified from Bryant and Fraser (1988). A glass capillary was filled with rhodamine-labeled dextran and introduced into an imaginal disc cell. The correct positioning of the capillary was followed by a small membrane potential between the capillary and a buffer drop (Hank's solution). A voltage impulse was applied to the system in order to drive the stain into the cell. The degree of injected stain was estimated visually under a fluorescence microscope.

\section{Culture and incision of the imaginal discs}

The in vivo culturing of wing imaginal discs was performed in the abdomens of adult females between one and five days old. The discs were dissected and injected into the abdomens in PBS supplemented with 100 $\mathrm{U}$ penicillin/ml and $0.1 \mathrm{mg}$ streptomycin $/ \mathrm{ml}$. The injected flies were maintained on standard medium and kept at $25^{\circ} \mathrm{C}$ during the culture period. 
Two different cuts were utilized (Fig. 1); incision removing tissue only from the anterior compartment denoted as $A B$ and incision disturbing both anterior and posterior compartments denoted as BC. The cultured discs were recovered from hosts after 24, 48, 72 or 96 hours and photographed with an AxioCam MRc digital camera attached to an Axiovert-200 fluorescence microscope. The discs showing well-preserved morphology and iontophoretic cell labeling were reinjected into hosts after photographing to reveal the dynamics of gene expression. The pictures obtained were merged and digitally processed using the Adobe Photoshop 5.5 software.

\section{Visualization of regenerative proliferation}

In order to reveal the specific location of regenerative proliferation, wing imaginal discs from wild type late third instar larvae were dissected, injected into adult hosts and cultivated for four days. This time period is sufficient to cease the disc's developmental proliferation and therefore to allow the observation of mitoses specifically induced by the regeneration process (Dale and Bownes, 1980; Bryant and Fraser, 1988). After the cultivation, the discs were recovered from the abdomens, cut with a tungsten needle (incision A'B, Fig. 1), photographed and reimplanted into fresh hosts for 48 hours. To visualize the mitotic cells of the recovered discs a H3-p primary antibody (rabbit anti-H3-p [Upstate Biotechnology]) was used (1:100 dilution). The following secondary antibody was FITC conjugated goat-antirabbit IgG, (Molecular Probes) (1:200 dilution). The discs were mounted in Mowiol supplemented with $10 \%$ DABCO and $0.2 \% \mathrm{NaN}_{3}$ and photographed with a Leitz Wild MPS46 camera attached to a Leitz Dialux 22 fluorescence microscope.

\section{Acknowledgements:}

This work has been supported by the Academy of Finland (Grant: 203530), by RFBR project 02-04-49323 and by the Turku University Foundation. We would like to thank Dr. Constantin Yanicostas for his critical comments and encouragement and also Dr. Graig Primmer for his assistance in the revision of the text. We are especially grateful to Bio-MedicalPhysics Chair of Novosibirsk University colleagues (headed by V.P. Maltsev) for the development of the iontophoretic device used in the study.

\section{References}

BECK, C.W., CHRISTEN, B. and SLACK, J.M.W. (2003). Molecular pathways needed for regeneration of spinal cord and muscle in vertebrate. Dev Cel/5: 429-439.

BROOK, W.J., OSTAFICHUK, L.M., PIORECKY, J., WILKINSON, M.D., HODGETTS, D.J. and RUSSELL, M.A. (1993). Gene expression during imaginal disc regeneration detected using enhancer-sensitive P-elements. Development 117: 12871297.

BRYANT, P.J. (1975). Pattern formation in the imaginal wing discs of Drosophila melanogaster. Fate map, regeneration and duplication. J Exp Zoo/193: 49-77.

BRYANT, P.J. and FRASER, S.E. (1988). Wound healing, cell communication, and DNA synthesis during imaginal disc regeneration in Drosophila. Dev Biol127: 197-208.
BURKE, R. and BASLER, K. (1996). Dpp receptors are autonomously required for cell proliferation in the entire developing wing. Development 122: 2261-2269.

CHEN, Y. and STRUHL, G. (1996). Dual roles for patched in sequestering and transducing hedgehog. Cel/87: 553-563.

DALE, L. and BOWNES, M. (1980). Is regeneration in Drosophila the result of epimorphic regulation? Wilhelm Roux's Archives 189: 91-96.

FRENCH, V., BRYANT, P.J. and BRYANT, S.V. (1976). Pattern regulation in epimorphic fields. Science 193: 969-981.

GAROIA, F., GUERRA, D., PEZZOLI, M.C., LÓPEZ-VAREA, A., CAVICCHI, S. and GARCÍA-BELLIDO, A. (2000). Cell behaviour of Drosophila fat cadherin mutations in wing development. Mech Dev 94: 95-104.

GIBSON, M.C. and SCHUBIGER, G. (1999). Hedgehog is required for activation of engrailedduring regeneration of fragmented Drosophila imaginal discs. Develop ment 126: 1591-1599.

GLISE, B. and NOSELLI, S. (1997). Coupling of Jun amino-terminal kinase and Decapentaplegic signaling pathways in Drosophila morphogenesis. Genes Dev 11: $1738-1747$

KUNIEDA, T., KURATA, S. and NATORI, S. (1997). Regeneration of Sarcophaga imaginal discs in vitro:implication of 20-hydroxyecdysone. Dev Bio/183: 86-94.

LECUIT, T. and COHEN, S.M. (1998). Dpp receptor levels contribute to shaping the Dpp morphogen gradient in the Drosophilawing imaginal disc. Development 125 : 4901-4907.

MILÉTICH, I. and LIMBOURGH-BOUCHON, B. (2000). Drosophila null s/mbclones transiently deregulate Hedgehog-independent transcription of wingless in all limb discs, and induce decapantaplegictranscription linked to imaginal disc regeneration. Mech Dev 93: 15-26.

NOSELLI, S. (1998). JNK signaling and morphogenesis in Drosophila. Trends Genet 14: $33-38$

O'BROCHTA, D.A. and BRYANT, P.J. (1987). Distribution of S-phase cells during the regeneration of Drosophila imaginal wing discs. Dev Bio/119: 137-142.

PODOS, S.D. and FERGUSON, E.L. (1999). Morphogen gradients: new insights from DPP. Trends Genet 15: 396-402.

RÄMET, M., LANOT, R., ZACHARY, D. and MANFRUELLI, P. (2002). JNK signalling pathway is required for efficient wound healing in Drosophila. Dev Biol 241: 145-156.

RIESGO-ESCOVAR, J.R. and HAFEN, E. (1997). Drosophila Jun kinase regulates expression of decapentaplegic via the ETS-domain protein Aop and the AP-1 transcription factor DJun during dorsal closure. Genes Dev 11: 1717-1727.

STRIGINI, M. and COHEN, S.M. (1997). A Hedgehog activity gradient contributes to AP axial patterning of the Drosophila wing. Development 124: 4697-4705.

TANAKA, E.M. (2003). Cell differentiation and cell fate during urodele tail and limb regeneration. Curr Opin Genet Dev 13: 497-501.

Received: January 2004

Reviewed by Referees: February 2004

Modified by Authors and Accepted for Publication: April 2004

Edited by: Christine Mummer 